\title{
IAPURDUM
}

Euskal ikerketen aldizkaria | Revue d'études basques |

Revista de estudios vascos | Basque studies review

$4 \mid 1999$

Numéro IV

\section{Le complexe verbal basque : un regard « universaliste »}

\section{Georges Rebuschi}

\section{OpenEdition}

\section{Journals}

Édition électronique

URL : http://journals.openedition.org/lapurdum/1553

DOI : 10.4000/lapurdum.1553

ISSN : 1965-0655

\section{Éditeur}

IKER

Édition imprimée

Date de publication : 1 octobre 1999

Pagination : 199-222

ISBN : 2-84127-156-0

ISSN : $1273-3830$

Référence électronique

Georges Rebuschi, «Le complexe verbal basque : un regard « universaliste »», Lapurdum [En ligne], 4 | 1999, mis en ligne le 01 avril 2010, consulté le 30 janvier 2020. URL : http://journals.openedition.org/ lapurdum/1553 ; DOI : 10.4000/lapurdum. 1553 


\section{Georges REBUSCHI}

\section{LE COMPLEXE VERBAL BASQUE : UN REGARD «UNIVERSALISTE »}

1. Dans cet article, je voudrais tenter de pousser jusqu'à ses limites une argumentation réduisant le grand foisonnement des formes conjuguées, simples et complexes, du basque à un schéma linéaire vieux aujourd'hui de plus de 40 ans, l'analyse du complexe verbal anglais proposée par Chomsky (1957). L'idée de base est la suivante : à un niveau relativement peu abstrait, le basque présente une image miroir de la structure du CV anglais, qui, si l'on fait abstraction de divers facteurs, s'analysait à l'époque comme en (1a) ci-après (certains symboles sont modifiés pour plus de clarté); plus spécifiquement, je souhaite montrer que le basque présente un CV comme celui de (1b) :

(1) a $C V=[$ AUX FLEX (M) (Aspect.1) (Aspect.2) $] \mathrm{V}$

b $\quad C V=V[$ AuX $(($ Aspect.2) Aspect.1) (M) FLEX]

Si (1b) n'est pas une image miroir parfaite de (la), c'est parce que la présence des morphèmes matérialisant l'aspect le plus proche du verbe lexical (Aspect.2) est subordonnée à la sélection du second (Aspect.1), alors que les marqueurs aspectuels complexes de l'anglais sont indépendants l'un de l'autre. Bien entendu, il y a beaucoup d'autres différences, plus ou moins superficielles ou importantes, comme le fait que FLEX en anglais correspond à un seul affixe marquant simultanément le temps et l'accord-sujet, alors que cet élément comporte jusqu'à trois marqueurs d'accord en basque, et que le temps en est dissocié, ou encore, inversement du point de vue de la richesse du matériel disponible, comme le fait que les Modaux de l'anglais représentent toute une classe de mots lexicaux autonomes, alors que le basque n'a que le morphème lié -ke à offrir; mais ces différences n'affectent en rien la profonde symétrie de (la) et (lb).

Cette symétrie est de plus, peut-être, l'expression d'une hiérarchie universelle, représentant, linéarisée de droite à gauche en anglais, et de gauche à droite en basque, une distance relative de plus en plus grande de l'aspect, puis de la modalité, et enfin du temps, par rapport au verbe lexical : à travers les langues, cette hiérarchie s'illustre aussi bien en français que dans diverses langues créoles, qu'elles aient un potentiel "substrat africain » ou non, comme l'a montré Bickerton (1981); et même si elle n'est pas universelle, j'espère que les considérations qui suivent contribueront à sortir la morphologie verbale basque de son exotisme : en particulier, on verra que le soi-disant « déplacement ergatif» ou D.E., qui a fait couler tant d'encre ces dernières années, n'est pas une transformation morphologique spécifique, mais simplement l'effet superficiel d'une règle de déplacement beaucoup plus générale, qui s'applique à certains affixes flexionnels basques, dont l'affixe absolutif - et à tous les affixes du CV anglais ou français ${ }^{1}$. 
Ce travail est organisé comme suit. En 2, je rappelle les motivations empiriques de l'analyse chomskyenne, ainsi que la transformation affixale T.A. (baptisée plus tard affix hopping) qui permet de passer des structures abstraites générées par (1a) à l'ordre linéaire concret des morphèmes entrant dans la composition des divers CV de la langue, et, en 3, je montre comment cette démarche s'applique au français, en soulignant que le premier segment qui suit FLEX en (1a), Modalité, y existe bien aussi. En 4, je propose une première version de la T.A. basque qui déplace vers l'initiale du premier verbe qui le précède l'affixe absolutif engendré sous FLEX en (lb). En 5 est élaboré le système aspectuel représenté dans la même formule. En 6, Ies divers paradigmes du passé (et de l'hypothétique) selon la personne (I, II vs. III) des actants sont présentés, en tant qu'ils déterminent, dans certains cas, l'apparition de préfixes ergatifs plutôt qu'absolutifs, ce qui conduit à exposer en 7 comment une T.A. généralisée doit être restreinte pour bloquer l'apparition d'un préfixe ergatif au présent lorsque l'argument absolutif est de 3e personne, et l'arg. ergatif, de le ou de $2 \mathrm{e} p$.; une comparaison est proposée entre l'analyse proposée ici et diverses versions de la transformation D.E. évoquée supra. En 8, les affixes datifs et allocutifs sont intégrés dans le système, et, en 9 , je montre qu'on peut considérer que le morphème modal -ke est bien un élément modal indépendant de la Flexion porteuse des marques de temps et d'accord, comme indiqué en ( $1 \mathrm{~b}$ ). Après une dernière (et très radicale) reformulation de la T.A. en 10, j'indique enfin en 11 comment l'analyse strictement linéaire développée jusqu'ici peut s'articuler à une représentation syntaxique, donc structurale ou hiérarchisée, de la structure de la proposition basque.

2. Dans Syntactic Structures (Chomsky 1957, dorénavant SS), on trouve une formule compacte, numérotée (28.III), qui permet de générer, c'est-à-dire de construire explicitement, les 48 « temps » simples et composés de l'anglais ${ }^{2}$ :

(2) $\mathrm{AUX} \rightarrow \operatorname{FLEX~(M)~(have+EN)~(be+ing)~}{ }^{3}$

"FLEX" remplace ici le symbole originel "C", et représente ce qu'on appelle aujourd'hui flexion (temps et accord); " $M$ " renvoie à la classe des auxiliaires modaux (will can, may, must et shall); have et be sont les auxiliaires aspectuels du parfait et du progressif, et $E N$ et ing représentent les suffixes des participes «passé » et "présent » qui leur sont respectivement associés (les petites majuscules de $1 x$ indiquent qu'il s'agit d'un élément qui a divers allomorphes). Les éléments entre parenthèses sont optionnels, mais quand deux éléments sont compris dans la même paire de parenthèses, on a un choix bloqué, par ex. celui de la présence simultanée de l'auxiliaire have du suffixe de participe passé - ce qui n'est qu'une autre façon de noter ce qu'on appelle aussi des morphèmes discontinus.

La notion de complexe verbal (CV) n'apparaît pas dans les textes de Chomsky, car ce n'est pas un constituant au sens ordinaire du terme; un CV se définit simplement comme la suite linéaire de tout le matériel qu'on trouve sous AUX, et du verbe lexical (d'où l'emploi du signe "=" plutôt que de la flèche de réécriture):

(3) $\mathrm{CV}=\mathrm{AUX}+\mathrm{V}$

Les formes participiales des éléments qui suivent have et be représentent intuitivement une sorte d'accord du verbe (auxiliaire ou lexical) sur leur droite; 
l'ordre concret ou superficiel des morphèmes s'obtient par application de la «transformation affixale » ou T.A. (4), où "\#" représente une frontière de mot $^{4}$ :

(4) $\mathrm{AF}+v \rightarrow v+\mathrm{AF}+\#$

(où " $v$ " désigne tant les verbes auxiliaires que les verbes lexicaux)

Ainsi, si l'on a sélectionné la séquence optionnelle $<b e+i n g>$ et le verbe walk, l'application de (4) donnera la suite $<$ be + walk + ing $+\#>$; si be, à son tour, était directement précédé de FLEX, une autre application de (4), à la séquence $<$ FLEX + be $>$ maintenant, donnerait $<$ be + FLEX $+\#>$ (concrètement is ou was selon le choix du temps, avec un sujet singulier - noter que l'irrégularité morphologique ne fait pas l'objet d'une étude particulière dans $S S$ ).

Un des résultats les plus intéressants de la description rappelée ici est la position de l'affixe FLEX, nécessairement en tête de la partie droite de (2) - car c'est seulement ainsi qu'on peut prédire son apparition sur le premier élément verbal des formes conjuguées après application de (4) : il s'ensuit que le verbe lexical n'est conjugué ou fléchi au sens strict (i.e. ne porte de marques de temps et d'accord) qu'en l'absence d'auxiliaire, et que les formes conjuguées simples sont en fait exceptionnelles, contrairement à l'idée reçue de la grammaire traditionnelle 5 .

3. Si l'on excepte le passé simple, qui ne relève pas de la catégorie énonciative du discours (Benveniste 1959), on peut adapter très aisément le système anglais au français. Ruwet (1968) l'a fait, mais d'une manière un peu insatisfaisante, comme on va le voir immédiatement. Cet auteur, après avoir éliminé le dernier choix bloqué de (2), en raison de l'absence de forme progressive en français, en élimine aussi l'élément $M$, arguant du fait que les verbes modaux de cette langue se comportent syntaxiquement comme des verbes ordinaires, lexicaux, prenant une proposition infinitive comme complément (comparer je veux/peux/dois chanter et j'aime chanter). S'il a raison sur ce point particulier, il a par contre tort, à mon avis, de nier l'existence d'une catégorie modale spécifique dans l'AUX français. En effet, comme on sait, le futur et le conditionnel sont respectivement formés par la suffixation des morphèmes flexionnels de présent (celui, irrégulier, d'avoir et d'aller) et de passé (de l'imparfait) à ce que l'on a coutume d'appeler, pour des raisons historiques évidentes, l'infinitif du verbe. Mais, en synchronie, il n'y a guère de raison de considérer que le $-r$ infinitif soit identique à l'affixe du futur et du conditionnel; d'une part, en effet, le premier a un allomorphe zéro (dans les verbes du premier groupe), ce qui n'est jamais le cas du second; d'autre part, on peut associer un signifié précis à ce $-r$ - de la conjugaison, celui de marquer la prédiction ${ }^{6}$, alors que ce sème n'est en rien associé à l'infinitif.?

En conclusion, il semble rationnel de proposer (5) comme contrepartie française de (1): ${ }^{8}$

(5) $\mathrm{AUX} \rightarrow \operatorname{FLEX}(\mathrm{r})(\sqrt{\text { avoir }}+\dot{E})^{9}$

Cette formule, qui demande également à être associée à la T.A. (4), appelle au moins deux commentaires. Tout d'abord, son intérêt n'est pas de rapprocher artificiellement les règles de construction du français et de l'anglais, mais de permettre de mieux saisir les causes de variation superficielle entre deux langues somme toute assez proches. Si l'on admet la description sémantique proposée pour -r-, et si le même sème est constitutif de l'emploi épistémique de will, des 
formes superficiellement aussi différentes que les conditionnels passés de (6) se laissent ramener à une toute petite différence morphologique entre les deux langues.

(6) a aurait marché

b would have walked

En effet, (6a) se décompose comme en (7a), qui n'est que le résultat de l'application de (4) à (7b), et (6b) se décompose comme en (8a), qui n'est à son tour que l'output de l'application de (4) à (8b) :

(7) a au $+\mathrm{r}+\mathrm{ait}+\#$ march $+\dot{E}+\#$

b ait $+\mathrm{r}+\sqrt[V]{ }$ avoir $+E+$ march-

(8) a woul $+\mathrm{d}+\#$ have $+\#$ walk + ed $+\#^{10}$

b $\mathrm{d}+$ will + have $+E N+$ walk

Si l'on compare les formes sous-jacentes (7b) et (8b), on voit que les deux constructions sont très proches : aux signifiants près, la seule différence est que le marqueur de prédiction est un affixe en français, alors que c'est un mot autonome en anglais.

L'autre remarque appelée par (5) concerne la place relative de FLEX et (r). Il est acquis aujourd'hui que les règles syntaxiques n'affectent pas la structure interne des mots. Dans (7b), (4) s'applique donc d'abord à la séquence $\langle r+\sqrt{a v o i r}\rangle$, donnant la forme (légèrement irrégulière) $[a u+r(+\#)]$, qui est un $v$, puisque avoir est un $v$; c'est donc ce $v[a u+r]$ qui est l'élément concerné par la seconde application de (4), l'affixation de $-r$-ayant rendu le radical $a v$-invisible aux processus syntaxiques $^{11}$. Si l'ordre de ces deux éléments avait été l'inverse, $-r$-précédant plutôt FLEX, on aurait dû violer le principe de non-accessibilité syntaxique de la structure interne des mots pour retrouver les formes « réelles ».12

4. Il est temps de passer au basque. Considérons d'abord les cas les plus simples. Quand un verbe est conjugué synthétiquement, c'est lui qui porte les marques de flexion (temps et accord). Mais quand il est conjugué périphrastiquement, ces marques sont sur l'auxiliaire ${ }^{13}$ :

(9) a n.oa/nind.oa.n

b j.oa.n na.iz/j.oa.n nint.ze. $n^{14}$

c j.oa.(i)ten na.iz/j.oa.(i)ten nint.ze.n

d j.oa.nen na.iz / j.oa.nen nint.ze.n

(10) a na.uka.zu/ nind.uka.zu.n

b e.duki na.u.zu

b' e.duki.tzen na.u.zu

b" e.duki.ko na.u.zu

c e.duki nind.u.zu.n

c' e.duki.tzen nind.u.zu.n

c" e.duki.ko nind.u.zu.n

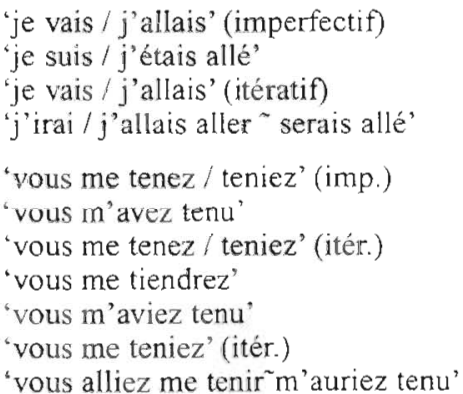

Rappelons la motivation du positionnement de FLEX à l'initiale de (2) : la marque de temps+accord ne peut se trouver sur le $v$ lexical (s'il n'y a pas d'auxiliaire), sur l'auxiliaire (s'il n'y en a qu'un), et enfin sur le premier auxiliaire (s'il y en a plusieurs), que si elle part de l'initiale du CV et est suffixée au $v$ qui la suit immédiatement. 
En basque, laissant les préfixes absolutifs un instant de côté, on peut dire de même en ce qui concerne la marque ergative, $-z u$ en (10), qu'elle est suffixée au $v_{\mathrm{L}}$ (verbe lexical), de racine - $u k a-$, en (a), où il n'y a pas d'auxiliaire, mais qu'elle est suffixée à l'auxiliaire de racine $-u$ - dans les cas (b-c). De manière symétrique par rapport à ce qui se passe en anglais et en français, on peut donc poser qu'il y a, en structure abstraite, un affixe "erg" initialement à droite (et suivi d'une marque temporelle, zéro au présent et $-(e) n$ au passé) qui se suffixe au $v$ qui le précède immédiatement - donc à l'aux. s'il y en a un, et au $v_{\mathrm{L}}$ autrement.

Quant au morphème absolutif, il présente la même distribution, à ceci près que c'est un préfixe : on le trouve donc à l'initiale de l'aux. s'il y en a un, mais à l'initiale du $v_{\mathrm{L}}$ autrement. Par parité de raisonnement avec ce qui précède, si l'on veut générer uniformément les cas de figure illustrés en (9) et (10), on est naturellement amené à proposer que cet affixe est situé abstraitement, ou « originellement ", à droite de la séquence $\left\langle v_{\mathrm{L}}\right.$ (...aux) $>$ : pour retrouver ce morphème en position de préfixe, il suffit de recourir à une T.A. symétrique de celle de l'anglais et du français, mais restreinte (temporairement) à la marque absolutive, à savoir :

(II) $v+a b s \Rightarrow a b s+v$

(où $v$ est à nouveau soit un aux. soit le $v_{\mathrm{L}}$ )

Comment concilier la suffixation de la marque ergative (et du morphème de temps) avec ce qui précède? Très simplement, en posant que la flexion comprend une séquence de trois affixes, "abs", "erg" et "TPS" (pour "temps") dans cet ordre :

(12) FLEX $\rightarrow$ abs (erg) + TPS

Comme l'entité abstraite AUX se présente (pour le moment) comme indiqué en (13), on a un CV de la forme (14), où ASP correspond à l'un des trois suffixes participiaux du perfectif, de l'imperfectif et du prospectif :

(13) AUX $\rightarrow$ (ASP + aux) FLEX FL $^{15}$

(14) $\mathrm{CV}=v_{\mathrm{L}}($ ASP + aux $)$ abs (erg) TPS

Dans (14), les seuls éléments obligatoires sont $v_{\mathrm{L}}$, "abs" et "TPS". Si l'on dérive un CV ne comportant que ces éléments, comme en (15a), l'application de (11) à cette séquence donnera $(15 b), c f$. (9a) - les séquences permutées sont en gras :

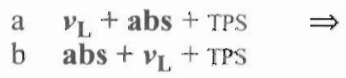

Si un affixe ergatif est également présent, partant de (16a), on obtient (16b), cf. (10a), c'est-à-dire qu'il n'est pas affecté par la T.A., et reste bien suffixé à $\nu_{\mathrm{L}}$ :

$$
\begin{array}{ll}
\text { a } & v_{\mathrm{L}}+\mathrm{abs}+\mathrm{erg}+\text { TPS } \\
\text { b } & \text { abs }+v_{\mathrm{L}}+\mathrm{erg}+\text { TPS }
\end{array} \quad \Rightarrow
$$

Les deux autres cas de figure correspondent à la sélection de (ASP + izan) dans (13), avec soit le seul affixe "abs" en FLEX, soit les deux marques d'accord "abs" et "erg". Le premier cas correspond à (17a) et le second à (18a); on vérifie qu'ils donnent, par application de la même T.A., (17b) et (18b) respectivement :

(17) a $v_{\mathrm{L}}+\mathrm{ASP}+\mathbf{a u x}+\mathbf{a b s}+$ TPS $\Rightarrow$

b $v_{\mathrm{L}}+\mathrm{ASP}+\mathrm{abs}+\mathrm{aux}+\mathrm{TPS}$

(18) a $v_{\mathrm{L}}+$ ASP + aux + abs + erg + TPS $\Rightarrow$

b $v_{\mathrm{L}}+\mathrm{ASP}+\mathrm{abs}+\mathrm{aux}+\mathrm{erg}+\mathrm{TPS}$ 
Par ailleurs, on a besoin d'introduire une frontière de mot \#, si l'on veut isoler ce qui est l'auxiliaire de surface; indépendamment des phénomènes phonologiques, dont certains sont contraints par la délimitation des mots, on sait que l'aux. est syntaxiquement autonome : il se préfixe à la marque de négation ez, laissant le $v_{\mathrm{L}}$ sur sa droite. L'objet morpho-syntaxique \# doit donc être inséré au cours de la transformation affixale ${ }^{16}$, ce qui nous amène à modifier (11) comme en (19), et par suite à retranscrire (15b), (16b), (17b) et (18b) comme en (20) :

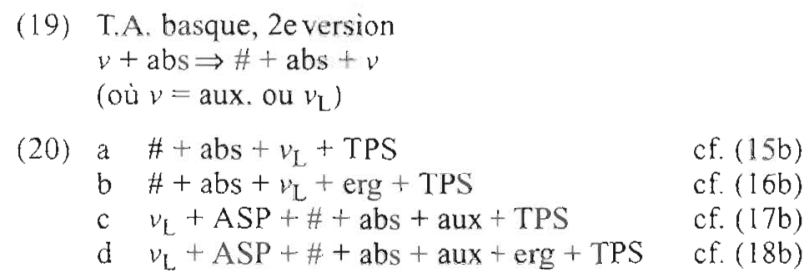

5. Avant de revenir à la structure interne de FLEX, considérons de plus près les séquences $<$ ASP + aux $>$ fournies par (13) supra. Il y a trois problèmes. Celui de la sélection d'un aux. intransitif ou transitif mimant la valence du $v_{L}$ peut se résoudre très facilement : il suffit d'exiger que le $v_{\mathrm{L}}$ et le (ou les : voir ci-dessous) aux. partagent le même trait [?transitif] (voir aussi la note 28).

Le second problème est celui des formes dites aoristiques. Il se présente comme suit : certains temps complexes offrent un radical verbal (plutôt qu'un participe) lorsqu'ils sont associés à un auxiliaire supplétif ( ${ }^{\circ} e d i n$ au lieu de izan intransitif, ${ }^{\circ}$ ezan au lieu de ${ }^{\circ}$ edun transitif) permettant de construire les potentiels, les subjonctifs et certaines formes hypothétiques (conditionnantes et conditionnées) ${ }^{17}$, cf. par exemple :

$\begin{array}{ll}\text { a } & \text { eduk na.za.zu.n } \\ \text { b } & \text { eduk na.za.ke.zu } \\ \text { c } & \text { edul nint.za.ke.zu.n } \\ \text { d } & \text { eduk ba-na.za.zu } \\ \text { e } & \text { eduk ba-nint.za.zu } \\ \text { f } & \text { eduk ne.za.ke.zu } \\ \text { g } & \text { eduk nint.za.ke.zu }\end{array}$

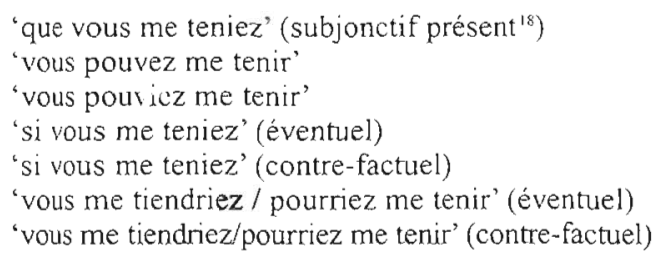

Descriptivement, il n'y a pas de difficulté (on reviendra sur les formants internes à l'aux.); il suffit de se donner la règle de réécriture (22) comme cxpansion d'ASP :

$\mathrm{ASP} \rightarrow\{T L(K O) / T Z E N / \emptyset\}$

Évidemment, le suffixe perfectif concret est phonologiquement variable : $-\varnothing$, $-i,-n,-t u$, mais, dans l'esprit de $S S$, je laisse ces variations de côté, de même que le choix entre -ten, -iten et -tzen pour l'imperfectif, ou encore en -(r)en et -ko pour le prospectif. Par contre, comme le participe prospectif se dérive toujours du perfectif, par suffixation de $-k o$ ou $-e n$, il est inutile de proposer deux choix distincts $\{T U / T U K O / \ldots\}$. On peut de même laisser la morphologie « concrète » décider de la forme du radical de l'aux. en fonction du préfixe aspectuel qui le précède (choix entre -eza- et - $u$ - pour les constructives transitives par exemple), selon que le suffixe est, respectivement, $\emptyset$ ou l'un des autres. 
Le troisième problème est celui de la reduplication de l'aux. dans des exemples comme :

$\begin{array}{ll}\text { a } & \text { eduki izan nauzu } \\ \text { b } & \text { eduki izaten nauzu } \\ \text { c } & \text { eduki izango nauzu } \\ \text { d } & \text { eduki izan }{ }^{21} \text { nazakezu }\end{array}$

'vous m'avez eu tenu' / 'vous m'avez souvent tenu'ts

'vous me tenez' (itératif) ${ }^{20}$

'vous me tiendrez' (itératif)

'vous pouvez me tenir' (itératif)

Noter ici que la variation aspectuelle ne peut se faire que sur le premier aux., et non sur le $v_{\mathrm{L}}$, comme le montre l'agrammaticalité des ex. suivants :

(24) a *edukitzen izan nauzu

b *edukiko izan nauzu

La présence d'un aux. participial entre le $v_{\mathrm{L}}$ et l'aux. proprement fléchi est donc conditionnée par le choix préliminaire de la séquence $<\mathrm{ASP}+\mathrm{aux}>$ dans AUX; l'aux. dupliqué (linéairement le premier en basque), est lui-même toujours précédé de l'affixe participial perfectif, noté $T U$. On est ainsi amené à modifier (13) comme suit (cf. (lb)) $)^{22}$ :

$$
\mathrm{AUX} \rightarrow((T U+\mathrm{aux}) \mathrm{ASP}+\mathrm{aux}) \text { FLEX }
$$

6. Revenant au composant "FLEX" de la catégorie AUX, regardons maintenant les corrélats morphologiques de son élément "TPS" (cf. 12). Pour commencer, contrairement à ce qui a été sous-entendu jusqu'ici, le basque n'a pas deux " temps » morphologiques, mais trois. En effet, à côté du présent et du passé, on a ausi des formes hypothétiques (dont la valeur correspond grosso modo à celle de l'emploi dit modal du prétérit anglais et de l'imparfait français). La différence entre les FVF (formes verbales fléchies) est maximalement visible lorsque tous les arguments du $v_{\mathrm{L}}$ sont de 3 e personne :

$\begin{array}{lllll}\text { a da } & \text { 'il est' } & \text { a' } & \text { da.tor } & \text { 'il vient' } \\ \text { b zen } & \text { 'il était } & \text { b' ze.torr.en } & \text { 'il venait } \\ \text { c ba-li.tz } & \text { 's'il était' } & \text { c' ba-le.tor } & \text { 's'il venait' } \\ \text { d li.tza.ke } & \text { 'il serait' } & \text { d' le.tor.ke } & \text { 'il viendrait' } \\ \text { a d.u } & \text { 'il (l')a' } & \text { a' } & \text { da.uka } & \text { 'il (le) tient } \\ \text { b z.u.en } & \text { 'il (l')avait' } & \text { b' ze.uka.n } & \text { 'il (le) tenait' } \\ \text { c ba-l.u } & \text { 's'il (l')avait' } & \text { c' ba-le.uka } & \text { 's'il (le) tenait' } \\ \text { d l.u.ke } & \text { 'il (l')aurait' } & \text { d' le.uka.ke } & \text { 'il (le) tiendrait' }\end{array}$

L'identification des préfixes $d(a)$-, z(e)-et l(e)-comme marqueurs d'accord ou comme marqueurs de temps est une question délicate, qui sera reprise dans la section suivante. Pour le moment, il suffit, comme je l'ai fait ci-dessus, d'associer globalement les FVF à des structures argumentales particulières. On reviendra également sur la segmentation effectuée en (26-27) et (28-29) ${ }^{24}$

Lorsque l'argument unique ou les divers arguments sont seulement de le et de 2 e personne, la différence entre les deux temps non-présents, ou non-actuels, est plus délicate : seule l'absence du -(e)n final permet de dire que l'on a pas affaire à un passé proprement dit, car le morphème $b a$ - 'si' des lignes (c) est compatible avec les trois temps, et le morphème -ke des lignes (d), sur lequel on reviendra en 9, l'est aussi. Les ex. de (28) et (29) résument la situation : 


$\begin{array}{ll}\text { a nint.z.en } & \text { j'étais' } \\ \text { a' nen.torr.en } & \text { 'je venais' } \\ \text { b ba-nint.z } & \text { 'si j'étais' } \\ \text { b' ba-nen.tor } & \text { 'si je venais' } \\ \text { c nint.za.ke } & \text { 'je serais / pourrais être' } \\ \text { c' nen.tor.ke } & \text { 'je viendrais/pourais venir' } \\ \text { a hind.u.gu.n } & \text { 'nous t'avions' } \\ \text { a' hind.uka.gu.n } & \text { 'nous te tenions' } \\ \text { b ba-hind.u.gu } & \text { 'si nous t'avions' } \\ \text { b' ba-hind.uka.gu } & \text { 'si nous te tenions' } \\ \text { c hind.u.ke.gu } & \text { 'nous t'aurions' } \\ \text { c' hind.uka.ke.gu } & \text { 'nous te tiendrions' }\end{array}$

Les préfixes absolutifs sont donc les mêmes au passé et à l'hypothétique. Cependant, ils n'ont pas la même forme que ceux du présent - voir les deux premières colonnes de (32) infra.

Passons maintenant au troisième cas de figure, qui se présente lorsque l'argument absolutif est de 3epersonne, mais qu'il y a aussi un argument ergatif de 1e ou de 2epersonne. On obtient des formes comme les suivantes :

$$
\begin{array}{ll}
\mathrm{a} & \text { n.u.en } \\
\mathrm{b} & \text { h.u.en } \\
\mathrm{c} & \text { gen.u.en } \\
\mathrm{d} & \text { zen.u.en }
\end{array}
$$

'je l'avais'
'tu l'avais'
'nous l'avions'
'vous l'aviez'

$\begin{array}{ll}a^{\prime} & \text { ba-n.u } \\ \text { b' } & \text { h.u.ke } \\ \text { c' } & \text { ba-gen.u } \\ \text { d' } & \text { ren.u.ke }\end{array}$

'si je l'avais'

\begin{tabular}{|c|c|}
\hline a ne.uka.n & 'je le tenais’ \\
\hline ba-he.uka & 'si tu le tenais' \\
\hline ba-gene.uka & 'si nous le tenions' \\
\hline zene.uka.ke & 'vous le tiendriez' \\
\hline
\end{tabular}

'tu l'aurais'

'si nous l'avions'

On a maintenant un préfixe ergatif. L'analyse de ce phénomène se fera dans la section suivante. Pour le moment, nous pouvons nous contenter du tableau (32), qui montre que, pris globalement, ces préfixes erg. sont différents des deux types de préfixes abs. :

(32 Préfixes des personnes I et II

\begin{tabular}{llcc}
\multicolumn{2}{c}{$a b s$, présent } & $a b s$, non-actuel & erg, non-actuel \\
Isg & na- & nind-2s & n(e)- \\
$2 \mathrm{sg}$ & ha- & hind- & h(e)- \\
$1 \mathrm{pl}$ & ga- & gind- & gen(e)- \\
$2 \mathrm{pol}^{26}$ & za- & zind- & zen(e)- \\
$2 \mathrm{pl}$ & za-...-te & zind-...te & zen(e)...te
\end{tabular}

On a évidemment envie de segmenter ces formes, car la consonne initiale marque clairement la personne (au sens où "lsg" et "lpl" sont deux personnes différentes, plutôt que la «même » personne associée à un nombre donné) $)^{27}$. La question qui se pose est donc de savoir s'il faut considérer que nous avons affaire à deux préfixes, l'un donnant exclusivement la personne, et l'autre indiquant simultanément (i) le cas de l'argument correspondant et (ii) le temps de la FVF, ou si au contraire nous n'avons qu'un seul préfixe, indiquant à la fois la personne, le cas et le temps. Comme la T.A. du basque, quelle que soit sa forme ultime, serait beaucoup plus difficile à formuler si l'on devait déplacer deux préfixes, dont le premier serait exclusivement personnel, et comme la forme même du second (-ind-vs. (e) ${ }^{\sim}$-en(e)) dépend non seulement du temps mais aussi de la 
fonction du préfixe précédent, j'adopterai la solution d'un préfixe unique, en proposant la règle (33):

(33) La valeur spécifique [ \pm présent] de l'élément TPS est copiée sur l'affixe déplacé par T.A.

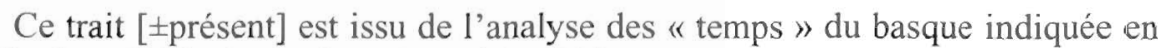
(34), dont (34') n'est qu'un résumé intuitif :

$\begin{array}{llll}\text { a } & \text { TPS } & \rightarrow & {[ \pm \text { présent }]} \\ \text { b } & {[- \text { présent }} & \rightarrow & {[ \pm \text { passé] }} \\ \text { c } & {[- \text { pré., -pas.] }} & \rightarrow & {[+ \text { hypoth.] }} \\ \text { d } & {[+ \text { présent] }} & \rightarrow & {[- \text { hypoth.] }}\end{array}$

(34') TPS $\rightarrow$ \{présent / passé / hypoth.

En ce qui concerne la forme phonologique concrète des affixes, un préfixe absolutif marqué [ + présent] serait donc sélectionné dans la première colonne de (32), et un préfixe abs. [-prés.] serait pris dans la seconde.

7.1. Les phénomènes décrits ci-dessus débordent largement la capacité d'application de la T.A. telle qu'elle est formulée en (19), puisqu'un affixe ergatif peut parfois être déplacé vers la tête de la FVF. Imaginons que le basque ne possède que des affixes de le et de 2e personne. L'interprétation des arguments alors nonreprésentés dans la flexion se fait sur la base de la valence inhérente des verbes fléchis basques : en effet, comme l'a remarqué de Rijk (1985), tous les verbes fléchis sont marqués comme transitifs ou intransitifs (ou plus précisément « inaccusatifs $\gg$ ). L'absence de suffixe sur des FVF comme doa ou dator s'interprète donc comme une absence d'argument ergatif ('il va', 'il vient'), alors que la même absence de suffixe sur des verbes fléchis comme dauka ou dakar s'interprète forcément comme renvoyant en syntaxe à un argument ergatif de 3ep. sg. ("il le tient', 'il le porte') : contrairement aux formes périphrastiques qui, associées à l'aux. monovalent $d a$, peuvent avoir une lecture médio-passive (edukitzen da 'il se tient/est tenu', ekarriko da 'il sera apporté'), les formes synthétiques citées à l'instant ne peuvent absolument pas s'interpréter de cette manière ${ }^{28}$.

Dans ces conditions, on pourrait remplacer (12) par (35) et (19) par (36), où $v$ représente toujours soit aux. soit $\nu_{\mathrm{L}}$, et où "aff FLEX" est pour " affixe flexionnel $\gg »^{29}$ :

(35) FLEX $\rightarrow$ (abs) (erg) TPS

(36) TA basque, $3^{e}$ version

$v+\operatorname{aff}_{\mathrm{FLEX}} \Rightarrow \#+\mathrm{aff}_{\mathrm{FLEX}}+v$

Si l'argument abs. est de le ou 2epers., il y a un affixe "abs" qui lui correspond dans FLEX, et c'est lui qui est déplacé parr (36), comme il l'était par (19). Si l'argument abs. est de 3epersonne, et qu'il y a un arg. ergatif de 1e ou de $2 \mathrm{e}$ pers., c'est maintenant ce dernier qui va pouvoir être déplacé (cas du passé et de l'hypothétique). Enfin, s'il n'y a pas d'argument ergatif, ou seulement un arg. erg. de 3ep., c'est la marque de temps (d(a)-, z(e)- ou l(e)-, cf. (26) et (27)) qui, en tant que premier affixe flexionnel, va passer en tête.

Malheureusement, ce système est trop puissant, car il permet aussi, contrairement aux faits, de faire passer un affixe ergatif en tête au présent, dès lors que 
l'arg. absolutif est de 3 e personne, ce qui donnerait par ex. ${ }^{*} g$ ? $u$ au lieu de $d . u$.gu 'nous l'avons' (le point d'interrogation marque la place qu'occuperait le morphe de temps qui apparait dans (32)). Comment bloquer cette surgénération?

Une solution est d'intégrer le $d(a)$-initial des formes de présent à abs. de $3 \mathrm{e}$ pers. (cf. (26a,a') et (27a,a') dans le paradigmes des affixes personnels. (35) et (36), qui sont maximalement généraux, restent inchangés, mais sont accompagnés de la restriction suivante :

(37) Dans la flexion verbale basque, il ñ'y a de marqueur d'ergatif de 3epersonne à aucun temps, et de marqueur d'absolutif qu'au présent.

Par rapport aux scénarios décrits à la suite de (36), le seul changement concerne donc le cas où, le temps étant le présent, il y a un argument syntaxique de 3epers. : on aura, en vertu de (37), un affixe absolutif dans la flexion, et c'est donc lui qui sera déplacé par (36). Une partie du contenu de (37) est effectivement stipulative, mais le fait qu'il n'y ait pas de préfixe ergatif au présent lorsque l'argument abs. est de 3e personne, et l'arg. erg., de leou de 2 e p., doit de toute manière faire l'objet d'un traitement spécial - à moins de considérer que c'est le « déplacement ergatif » qui est irrégulier : c'est ce que postulent toutes les descriptions faites jusqu'ici (cf. 7.2 infra), mais, hors de tout cadre théorique, rien ne permet de dire vraiment si c'est d.u.gu 'nous l'avons', avec un suffixe ergatif, ou gen.u.en, avec un préfixe ergatif, qui est régulier, ou irrégulier. Par ailleurs, si l'on s'étonne de la disparité de traitement entre les marqueurs d'accord absolutif et ergatif, on prendra note de ce que les accords de datif, qui seront examinés en 8 , diffèrent des deux autres par le fait qu'il existe bien un suffixe de datif de $3 e p$. sg., -o, à tous les temps. Qu'il y ait donc des différences entre les trois types d'affixes personnels représentés dans les FVF, "abs", "erg" et "dat", et pas seulement entre le troisième et les deux autres, ne devrait donc pas être en soi particulièrement surprenant.

7.2. Quoi qu'il en soit, le système adopté ici présente plusieurs avantages sur la théorie du « déplacement ergatif $»^{30}$ ou D.E. qui est à l'heure actuelle le traitement le plus répandu des incohérences ou irrégularités du positionnement des affixes constatées dans la section 6. Pour commencer, la théorie du D.E. n'est pas générale, car elle se restreint à un seul cas de déplacement affixal, qu'elle présente de plus comme une irrégularité : en fait, ses défenseurs admettent sans discussion, et contrairement à l'analyse proposée ici, que la marque d'abs. est pour ainsi dire originellement en position initiale de la FVF. On devine alors les difficultés qui vont surgir quand on voudra rendre compte explicitement de la répartition de cet élément sur le second aux. quand il y en a deux, l'unique aux. s'il n'y en a qu'un, ou enfin le $\nu_{L}$ s'il n'y a aucun aux.; en fait, personne n'a, que je sache, essayé de décrire ces questions de manière homogène jusqu'ici.

Mais il y a plus grave : en postulant que le préfixe abs. est " naturellement » un préfixe, on stipule par-là méme que la position initiale de la FVF est une position absolutive. En d'autres termes, si l'on admet la théorie D.E., il faut dire ou bien qu'un affixe identifié comme ergatif vient occuper une position vide identifiée elle-même comme absolutive, ce qui viole toutes les contraintes connues sur l'homogénéité des propriétés des éléments déplacés et de leur site d'accueil, ou bien que l'affixe déplacé " devient » lui-même un affixe absolutif, ce qui est rationnellement encore moins engageant. 
La première difficulté, déjà remarquée par Manandise (1988: 226) est illustrée par la description suivante du D.E. par Rotaetxe (1998: 219):

"Règle de DE : Aux formes du passé, d'abs. 3 et d'erg. 1 ou 2, l'indice actanciel de l'ergatif est déplacé vers la case vide de l'absolutif, prenant la forme indicielle de ce dernier, mais conservant sa coréférence avec un [argument] ergatif du verbe." [Les italiques sont de moi, G.R.] $]^{31}$

Quant à la seconde, elle transparaît dans le passage suivant d'Albizu (1997 : 8), qui, comparant les ex. $(38 \mathrm{a}, \mathrm{b})$ et $(39)$ - ses $(11 \mathrm{a}, \mathrm{b})$ et $(12)$ - ci-après, écrit :

"L'exemple $[(39)]$ avec un verbe inaccusatif est introduit pour montrer que le préfixe $z$ - de (38b) est en fait un marqueur absolutif." [Italiques de moi]

(38) a Zuk Anderri... sarrera bat oparitu d-i-o-zu

vous-ERG Ander-DAT billet un offert abs3sg-AUX dat3sg-erg2

'vous avez offert un billet à Ander'

b Zuk Anderri... sarrera bat oparitu $z$-eñi-o-n

vous-ERG Ander-DAT billet un offert ABSERG 2 sg-AUX-dat3sg-passé en $^{32}$

'vous aviez offert un billet à Ander'

(39) $\mathrm{Zu}$ berandu iritsi z-ara bilerara

vous-ABS en retard arrivé abs2-AUX réunion-la-à

'Vous êtes arrivé en retard à la réunion'

Ces raisonnements sont d'autant plus étranges qu'ils conduisent à une segmentation des verbes fléchis soit gênante (dans le premier cas), soit radicalement fantaisiste (dans le second). En effet, contrairement à ce que dit Rotaetxe, les préfixes personnels ne sont pas les mêmes selon que les préfixes sont absolutifs ou ergatifs, cf. (32) supra et la discussion qui suit ce tableau. En ce qui concerne la description proposée par Albizu, par contre, elle revient à transformer la racine de l'aux., qui passe de $-i$ - dans (38a) à -eni-dans (38b), ce qui exigerait pour le moins une argumentation sérieuse ${ }^{33}$ !

7.3. Revenons un instant sur les marqueurs du passé. Du point de vue segmental, on peut penser qu'il y a peut-être deux segments ou morphes correspondant au passé, l'un, z-, qui n'apparaît que si le morphème de temps est soumis à la T.A., et l'autre, le suffixe -(e)n, qui est présent quel que soit l'affixe qui passe à gauche du $v$ qui précède. On peut alors se demander pourquoi l'hypoth. ne possède pas de suffixe particulier - ni le présent non plus, qui n'apparaît jamais comme préfixe isolé d'ailleurs, si l'hypothèse selon laquelle le préfixe $d(a)$ - est bien un morphème d'abs.3.sg (spécifié pour ce temps par la règle (33)) est juste.

Ce dédoublement pourrait être réduit par le raisonnement suivant : il est bien connu que le biscayen, qui présente un préfixe de passé $\varnothing$ - plutôt que $z$-, reflète probablement un état de langue plus ancien; le basque commun aurait généralisé le $z$ - initial de zen 'il était', qui faisait originellement partie de la racine $(i . z a . n)^{34}$; pour l'analyse proposée ici, que l'on ait un $\varnothing$ - initial pour le passé ou un z- initial est indifférent, l'essentiel étant que l'on ait une marque de temps passé comme préfixe dans les conditions décrites.

Noter cependant que l'idée d'un $\varnothing$ - antique va de pair avec celle, généralement admise, qui consiste à dire que la marque fondamentale de passé est le suffixe -(e)n que nous avons rencontré plusieurs fois : le préfixe $z$-ne ferait que dupliquer d'une certaine manière l'information fournie par ce suffixe. Mais on peut objecter qu'en fait, ce suffixe n'est pas forcément temporel, puisqu'il se 
confond avec le suffixe de subordination des relatives, du subjonctif (cf. (2la)) et des questions indirectes, et qu'il disparaît en présence du suffixe de subordination des complétives assertives - (e)la. De plus, la question la présence du circonfixe $z-\ldots$ (e) n n'est toujours pas résolue.

Par ailleurs, il faudrait rendre compte du haut-navarrais méridional, qui, depuis qu'il est attesté ${ }^{35}$, a toujours fourni des formes verbales passées sans suffixe temporel, mais qui a bien un préfixe $z$ - dans les conditions requises. Qui plus est, l'ancien biscayen, très éloigné géographiquement du haut-navarrais, a aussi connu des passés sans $-n$ final (mais avec malgré tout un - $e$ ou un $-a$ qui n'appartient pas au radical!), comme le montrent les proverbes suivants tirés des Refranes y Sentencias anonymes de $1596^{36}$, dont Lakarra (1996) est une édition récente (on notera aussi qu'un $z$-, orthographié $c$-, est présent dans la FVF du second fragment, dont l'argument abs. est de 3e pers.) :
(40) a
Adiunça onean nentorre, vaya ezer ez necarre occasion bonne-loc je-vins, mais rien NEG j'apportai
"En buena sazón vine, mas no truxe nada"
b Arzayoc arri citea, gaztaeoc aguir citea
bergers $\sqrt{ }$ s'étonner AUX, fromages $\sqrt{\text { apparaître }} \mathrm{AUX}^{37}$
"Rinieronse los pastores de ovejas, y parecieron los quesos"
$\left(n^{\circ} 332\right)$
(n³86)

On peut donc penser que le basque ancien offrait deux possibilités quand l'absolutif était de 3 e pers. : soit une préfixation en $\varnothing$-, accompagnée du suffixe - $(e) n$, soit une préfixation en $z$-, mais sans suffixation d'accompagnement. Pour rendre compte du basque standardisé (et commun, transdialectalement), on pourrait donc avancer l'idée suivante (qui sera reprise dans un autre contexte en 9.1) : étant donné que les déplacements s'analysent, depuis Chomsky (1995) comme une double opération, d'abord de copie d'un élément $\alpha$ dans une autre position, puis d'effacement d' $\alpha$, le « déplacement » de l'affixe "passé" est en fait une copie, non suivie de l'effacement du segment d'origine; une forme comme z.u.en 'il avait' (de même que le biscayen $\varnothing$.u.an correspondant) serait donc la matérialisation de cette double présence du morphème en question : $<\#+$ passé $+\sqrt{ } v_{\text {AUX }}$ + passé $\alpha>$, sans, bien sûr, aucun affixe personnel.

8.1. Le CV basque contient encore trois affixes. Les deux premiers, qui sont à nouveau des marques personnelles, vont être analysés dans cette section. Le premier d'entre eux apparaît dans les ex. empruntés à Albizu plus haut; il s'agit de la marque d'accord datif : quand une FVF contient un tel élément, ce dernier est plus proche du radical que le suffixe ergatif, comme le montrent diozu dans (28a) et l'aux. dizugu dans (4l):

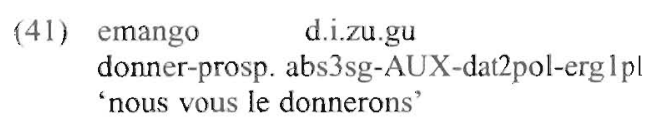

Le problème soulevé par cet affixe est le suivant : si un morphème d'accord "dat" (pour datif) est introduit à gauche de "erg" dans (35), la règle (36) prédit à tort que c'est cet affixe qui deviendra préfixe dans la version de (41) au passé, i.e. dans (42a), alors que c'est encore le marqueur d'ergatif qui se préfixe, comme indiqué en (42b) : 
(42) a

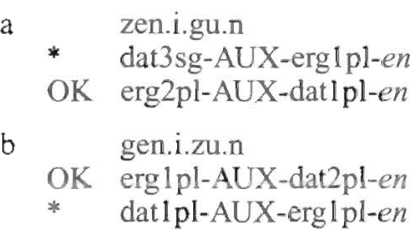

La difficulté provient de l'idée que l'affixe "dat" à insérer dans la version modifiée de (28) devrait suivre, plutôt que précéder, l'affixe "abs". Supposons par contre que l'élément optionnel "dat" y précède "abs", comme en (43), et que (36) soir modifié en conséquence comme en (44):

(43) FLEX $\rightarrow$ (dat) (abs) (erg) TPS

(44) T.A. basque, 4eversion

$[\nu($ dat $)] \mathrm{aff}_{\mathrm{FLEX}} \Rightarrow \#+\mathrm{aff}_{\mathrm{FLEX}}+[v+($ dat $)]$

On préserve alors la position suffixale du marqueur d'accord datif, ce qui permet la prédiction suivante, effectivement correcte : lorsqu'une FVF correspond à un prédicat biactanciel impliquant un absolutif et un datif, même si l'argument abs. est de 3 e personne, il n'y a pas non plus de préfixe datif, cf. :
(45) a ha.tor.ki.t
abs2sg-V venir-DF-1sg ${ }^{38}$
a' ha.tzai.t abs2sg- $v_{v_{\mathrm{AUX}}}-\mathrm{DF}-1 \mathrm{sg}$
b' $\emptyset . z a i . t^{39}$
'il m'est'
prés. $-\sqrt{v_{\mathrm{AUX}}}-1 \mathrm{sg}$
c' zi.tzai.da.n

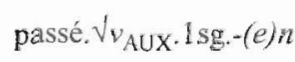
da.tor.ki.t
prés.- $\gamma_{\text {venir-DF-1sg }}$
'il vient à moi'
c ze.tor.ki.da.n

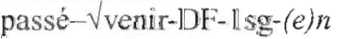
'il venait à moi'
'il m'était'

Noter à propos un nouvel avantage de mon analyse contre l'hypothèse D.E. : l'ordre des affixes dans (43) reflète directement la hiérarchie d'accessibilité à la fonction sujet : c'est l'élément le plus éloigné de $v$ qui en est le sujet ou «argument externe" » (i.e. l'argument ergatif, ou à défaut l'absolutif, mais normalement pas le datif), ce qui n'est finalement qu'un résultat fort banal ${ }^{40}$.

8.2. Un autre affixe personnel encore peut apparaître dans les FVF, qui ne se déplace pas non plus : c"est la marque allocutive, qui suit toujours immédiatement la marque dative ${ }^{41}$; comme dans la plupart des cas la consonne initiale de l'allocutif masculin $-k(a)$ - est élidée pour des raisons morphophonologiques indépendantes, je donnerai des exemples avec un interlocuteur féminin :

(46) a eman z.i.o.na.gu ${ }^{42}$

donné $d-\sqrt{ } v_{\mathrm{AUX}}$-df3sg-all2fém-erglpl

'nous le lui avons donné' (en s'adressant à une femme)

b eman gen.i.o.na.n

donné erglpl- $\sqrt{ } v_{\mathrm{AUX}}$-df3sg-all2fém-(e)n

Nous pouvons donc enrichir maintenant (43) et (44) comme suit :

(47) $\mathrm{FLEX} \rightarrow$ (dat) (ail) (abs) (erg) TPS

(48) T.A., 5e version

$[v($ dat $)($ all $)]$ aff FLEX $\Rightarrow \#+\operatorname{aff}_{\mathrm{FLEX}}+[v($ dat $)($ all $)]$ 
9.1. Passons au troisième affixe annoncé en 8.1. Les FVF basques comportent optionnellement un morphème -ke d'apport sémantique modal, qui couvre un domaine allant de la prédiction (cf. son emploi dans Ies formes hypothétiques des apodoses, i.e. conditionnées, comme en (27d) supra, ou comme marqueur de futur en souletin) à l'équipossibilité (formes fléchies dites potentielles), en passant par la probabilité (usage fréquent surtout dans les dialectes du nord autres que le souletin). En basque standardisé, dont la morphologie verbale est très proche du dialecte guipuzcoan, cet élément apparaît entre le suffixe datif et le suffixe allocutif (s'il y en a un). La question se pose donc de savoir s'il faut remplacer (47) par quelque chose comme (49) :

(49) FLEX $\rightarrow$ (dat) (ke) (all) (abs) (erg) TPS

Évidemment, dans cette position, on ne s'attend pas à ce que -ke soit déplacé par la version modifiée correspondante de (48) - et il ne se déplace effectivement pas -, mais il reste un problème conceptuel : que diable vient faire un affixe modal au milieu d'affixes de personne ${ }^{43}$ ? Si l'on revient à (47), on voit que la séquence des affixes est en fait composée de deux types bien différenciés, des affixes de personne, puis un affixe de temps. L'introduction du morphème modal -ke entre "dat" et "alloc" viendrait rompre cet arrangement régulier, sans parler du fait que la modalité épistémique ne rentre usuellement pas dans la catégorie de la flexion (temps/accord) ${ }^{44}$.

En fait, si l'on regarde la langue dans toute sa variété, tant dialectale que diachronique, on voit que le verbe unifié ou standardisé ne représente que l'une des deux options possibles. En consultant par exemple le relevé systématique des FVF du basque du XVI" siècle effectué par Lafon (1944), on voit qu'une écrasante majorité de formes contenant à la fois un affixe de datif et ce suffixe modal les présentent dans l'ordre inverse, <ke + "dat">. En voici quelques exemples (les deux premiers sont orientaux, et le troisième, occidental):

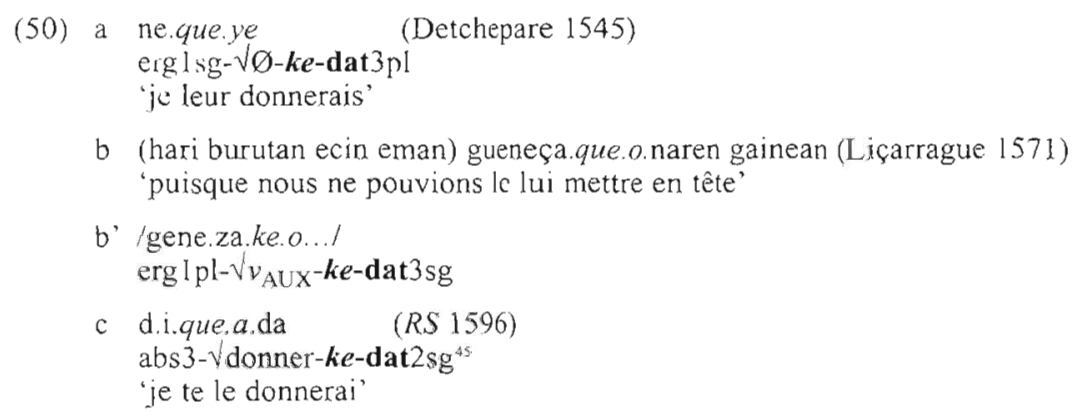

Au XIX siècle, Bonaparte (1869), recensant en particulier les formes des quatre dialectes dits littéraires, propose des tableaux dans lesquels, au potentiel comme au conditionnel, le morphème -ke précède toujours le suffixe datif en souletin et en biscayen, et ces données restent vraies aujourd'hui pour ces dialectes (voir Azkue (1923-25) pour le biscayen, et Mizpiratzéguy (1936) et Lüders (1993) pour le souletin) - mais l'affixe - ke, souvent réalisé ki- dans ces dialectes, n'est pas toujours visible, la séquence $<\sqrt{v_{A U X}}{ }^{+-k e}>$ tendant à être remplacée par un auxiliaire supplétif $(-a i-,-e i-,-i r o-)^{46}$. Les dialectes centraux, guipuzcoan et labourdin, offrent par contre l'ordre inverse dans les tableaux de Bonaparte, mais il a simplifié les données, du moins en qui concerne le second : Ithurry (1895), 
puis Arotçarena (1951), proposent les formes en <-ke + datif $>$ comme normales, signalant l'ordre inverse comme variante, tandis que Lafitte (1962) donne ces deux ordres comme équivalents.

De plus, en navarro-labourdin, il y a des préférences : quand l'affixe datif est de le ou 2ep., il semble suivre généralement -ke, mais le précéder quand il est de 3ep.; cependant, l'explication n'est probablement pas grammaticale, mais phonique : les affixes datifs de leet $2 \mathrm{e}$. commencent par une consonne $(-d(a),-k(a) /-n(a), g u, z u)$ alors que ceux de $3 \mathrm{ep}$. sont des voyelles ( $-o$ au sg., et $-e$ ou $-o+t e$ au pl.); on peut donc penser que le réagencement superficiel de $\langle k e+o>$ en $\langle o+k e>$ est dû à des raisons d'euphonie, le passage à droite de -ke permettant d'éviter un hiatus. Il aurait pu se généraliser plus au moins par la suite aux autres formes, sous l'influence probable du guipuzcoan, qui a manifestement été le premier dialecte à le faire.

A ce propos, il faut noter que, dans la grammaire manuscrite de P. d'Urte, qui décrit le labourdin du début du XVIII' siècle (Allières 1985), on relève des formes à redoublement de -ke-, comme gind.arrai.z.ké.tzu.ke 'nous vous suivrions' (p. 912), où l'affixe datif est " pris en sandwich » entre les deux occurrences de $-k e$. La signification de ces duplications est claire, si l'on admet (comme on l'a déjà fait dans un autre contexte en 7.3) que les "déplacements " sont en fait des copies suivies d'effacements : les formes " étranges 》 comme celle citée à l'instant sont en fait la trace de cette transformation, la copie ayant eu lieu de gauche à droite (si l'on tient compte des données du. XVI ${ }^{e}$ siècle), l'effacement n'ayant par contre pas été effectué. ${ }^{47}$

9.2. Il résulte de ce qui précède $q$ u'il y a un ordre sous-jacent $<k e+$ datif $>$, qui donne naissance à des FVC comme en (51), avec inversion ou réordonnancement purement superficiel de ces deux affixes en guipuzcoan (obligatoirement) et en labourdin (optionnellement) :

(51) $v+k e+$ dat $\ldots$

Ce que (51) apporte, c'est la possibilité d'introduire -ke indépendamment de la Flexion, comme dans (52), révision de (25), où cet affixe correspond à l'élément optionnel " $\mathrm{M}$ " :

(52) AUX $\rightarrow((T U+$ aux $)$ ASP + aux) (M) FLEX

Si un auxiliaire est sélectionné, le $v$ de (51) sera cet aux., et si aucun aux. n'est pris, ce $v$ sera le verbe lexical.

Il nous suffit donc de modifier encore une fois la formulation de la T.A., cf. (48), pour obtenir les FVF dotées de cet affixe modal spécifique, la composition interne de FLEX restant comme en (47):

(53) T.A. (6e version)

$[v(\mathrm{M})($ dat $)($ all $)]$ aff $_{\mathrm{FLEX}} \Rightarrow \#+\operatorname{aff}_{\mathrm{FLEX}}+[v(\mathrm{M})($ dat $)($ all $)]$

Une objection à mon analyse pourrait s'appuyer sur le fait que, dans les dialectes dans lesquels - ke-précède effectivement l'affixe datif (ou peut le précéder), il sépare cet affixe du morphème pré-datif "DF" lorsque ce dernier est présent ou visible, ce qu'illustrent les formes non-standardisées suivantes : 


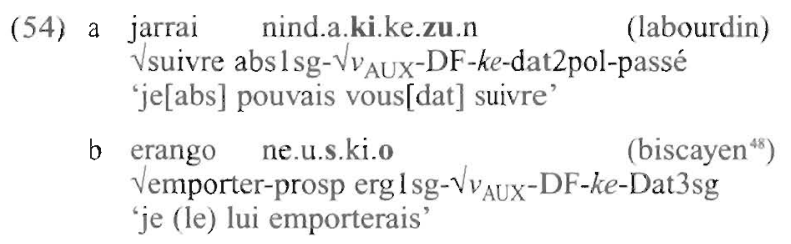

Faut-il, pour défendre (52) contre (49), recourir à un nouveau déplacement affixal? Ce serait une procédure ad hoc, mais une autre possibilité nous est offerte : on peut considérer que le marqueur pré-datif est en fait incorporé lexicalement au verbe, permettant ainsi une extension de sa valence (un peu à la manière des « prépositions » incorporées au verbe dans les langues bantu). Cet élément (-ki-, $-k-,-i-,-t s$ - selon les verbes et les dialectes) appartiendrait donc à la racine $v$, ainsi devenue complexe, qu'il s'agisse d'un $v_{\mathrm{LEX}}$ ou d'un $v_{\mathrm{AUX}}{ }^{49}$ En conséquence, il serait tout simplement invisible dans les processus qui nous concernent.

10. Récapitulons. Les résultats obtenus sont concentrés dans les règles (52), (22), (47), (34') et (53), répétées et renumérotées ici :

(55) $\mathrm{AUX} \rightarrow((T U+$ aux $)$ ASP + aux $)(M)$ FLEX

(56) $\mathrm{ASP} \rightarrow\{T U(K O) / T Z E N / \emptyset\}$

(57) FLEX $\rightarrow$ (dat) (all) (abs) (erg) TPS

(58) TPS $\rightarrow$ \{présent / passé / hypoth \}

(59) T.A. : [v (M) (dat) (all) $\operatorname{aff}_{\mathrm{FLEX}} \Rightarrow \#+\mathrm{aff}_{\mathrm{FLEX}}+[v$ (M) (dat) (all) $]$

(55) correspond bien à la formule ( $1 \mathrm{~b}$ ) présentée dans l'introduction : la spécificité du basque par rapport à des langues comme le français ou l'anglais ne tient donc pas tant à l'ordre dans lequel le verbe lexical et les marques aspectuelles, modales et flexionnelles de temps et d'accord se réalisent (il n'y a finalement que deux possibilités de linéarisation!) ni à la grande richesse de son élément FLEX (il existe bien des langues à conjugaison pluripersonnelle, même si celles à conjugaison tri-personnelles sont effectivement rares), mais surtout à la spécificité de la transformation affixale (59). C'est en effet le locus des «bizarreries » dont il a fallu rendre compte, et qui trouvent leur expression condensée dans la forme même de cette règle, puisque les premiers segments qui suivent $v$, même s'ils sont affixaux, ne subissent jamais le déplacement symétrique de la T.A. anglaise ou française.

Une hypothèse vient cependant à l'esprit, qui pourrait réduire encore cet « exotisme » des CV basques. On a vu en 3 , à propos des ex. (6a,b), que la différence superficielle entre les conditionnels passés de français et de l'anglais provenait somme toute très simplement d'une propriété des marqueurs de prédiction : en anglais, c'est un $v_{\mathrm{AUX}}$, alors qu'en français, ce n'est qu'un affixe. Dans le même esprit, en allant un peu plus loin dans le détail morphologique, on peut proposer que les affixes peuvent, à leur tour, être marqués ou non comme étant plus spécifiquement des préfixes ou des suffixes ${ }^{50}$. Admettons donc que les morphèmes qui correspondent à " $M$ " dans (55), et "dat" et "all" dans (57), soient spécifiés comme [+suffixes], ce qui est une donnée empirique évidente, et que les autres ne soient pas marqués. Au lieu d'utiliser les symboles "+" et "-", il faudrait 
plus précisément recourir à $M$ pour marqué, et $U$ pour non-marqué, sur le modèle de Chomsky \& Halle (1968). La T.A. basque se ramène alors à la règle (60):

(60) $[v(\mathrm{X})]$ aff $_{\mathrm{FLEX}}[$ Usuffixe $] \Rightarrow \#+$ aff $_{\mathrm{FLEX}}[$ Usuffixe $]+[v(\mathrm{X})]$ où $\mathrm{X}$ ne contient que des éléments [Msuffixes]

Le choix entre $M$ et $U$ (plutôt qu'entre + et -) se comprend comme suit : si les affixes susceptibles de se déplacer étaient explicitement marqués comme [-suff] ou [+préf], on construirait des FVF avec un préfixe effectivement en tête, mais de soi-disant préfixes (les affixes suivants) pourraient aussi apparaître en finale de mot! La solution adoptée ici permet d'éviter cette contradiction : les éléments non-marqués sont simplement identifiés comme préfixes s'ils ont été déplacés (s'ils suivent immédiatement " $\#$ "), et comme suffixes autrement. Ces propriétés sont ensuite directement exploitables dans l'épel phonologique des morphèmes. Par exemple, on tiendra compte de cette propriété de surface pour "lire " "erglsg" soit comme $n(e)-:$ si c'est un préfixe, soit comme $-t / d a-$ : si c'est un suffixe ${ }^{51}$.

11. Toute l'approche développée ici s'est faite en termes strictement linéaires: c'est en partie pour des raisons de simplicité dans la présentation, mais surtout parce que les approches structurales ou hiérarchiques, généralement inspirées par la « théorie X-barre », ou bien sont également influencées par des présupposés syntaxiques qui ne me semblent pas toujours justifiés, ou bien ont simplement fait l'économie d'une analyse aussi approfondie et cohérente que possible des données morphologiques. Cela dit, je pense qu'une reformulation de (55) et (57), ou plutôt des structures que ces règles génèrent, en arbres syntagmatiques est non seulement possible, mais même relativement simple, l'essentiel étant de se dire que (59) ou (60) sont des règles reflétant des propriétés morphologiques, et non des règles syntaxiques.

Considérons ainsi une dérivation qui n'aurait pris que FLEX dans (55), puis les éléments "dat", "abs", "erg" et TPS de (57). Il lui correspondra une architecture de phrase comme la suivante :

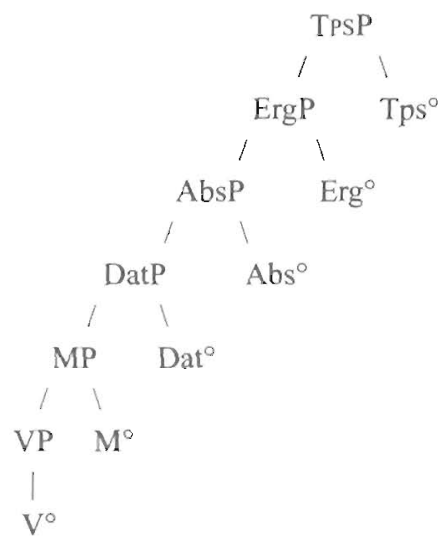

On y reconnaît fondamentalement un traitement classique du basque, selon lequel les têtes fonctionnelles sont à droite de leur complément. Ce qui est est moins classique, bien entendu, c'est l'ordre respectif des têtes. Comparer à cet 
égard la formulation due à Laka (1993, (22)), bien trop directement dépendante de la morphologie superficielle des formes du présent ${ }^{52}$ (pour faciliter la comparaison, je reprends mes propres abréviations) :

$$
\text { "abs" - v - "dat" - M - "erg" - TPS }
$$

Si des arguments sont associés à ces têtes, ils seront, dans les deux théories, dans la position des spécificateurs ${ }^{53}$.

Par ailleurs, les suites de morphèmes qui servent d'input à la T.A. s'obtiennent en deux temps. D'abord, il y aurait un déplacement successif de $\mathrm{V}^{\circ}$ dans $\mathrm{M}^{\circ}$, donnant $\left[\mathrm{V}^{\circ}+\mathrm{M}^{\circ}\right]$, puis de cet élément dans Dat ${ }^{\circ}$, de l'objet ainsi assemblé dans $\mathrm{Abs}^{\circ}$, etc., le tout aboutissant par exemple à la suite (63) :

$$
\left[\left[\left[\left[\left[V^{\circ}+\mathrm{M}^{\circ}\right] \mathrm{Dat}^{\circ}\right] \mathrm{Abs}^{\circ}\right] \mathrm{Erg}^{\circ}\right] \mathrm{TPS}^{\circ}\right]
$$

Cette tête fonctionnelle complexe serait ensuite convertie, au niveau dit de la Forme Phonique, en 1 (une des quatre suites de (64), en fonction des traits de personne de $\mathrm{Abs}^{\circ}$ et $\mathrm{Erg}^{\circ}$ (et/ou de leurs arguments), et de la contrainte (37) :

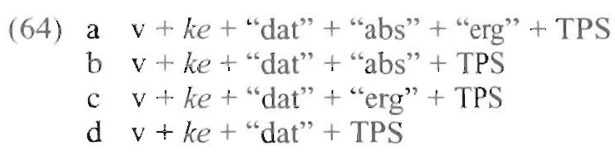

et ce seraient ces dernières suites qui subiraient véritablement la tran:formationaffixale. Il n'y a donc aucun obstacle de principe à une réinterprétation des résultats obtenus ici dans un formalisme explicitement syntaxique ${ }^{54}$, bien au contraire.

D'une part, cette interprétation de l'analyse morphologique développée au long de cet article permet de lever ce qui a dû apparaitre comme une contradiction, à savoir, que la règle T.A., qui est une règle morphologique, devrait parfois précéder l'application de règles syntaxíques, comme le déplacement de la FVF vers la position de la négation, qui est située plus haut que TPSP, mais sur sa gauche (Laka 1991). En fait, la T.A. va s'appliquer sous le nœud TPS ${ }^{\circ}$ de (61), étant donné le mouvement syntaxique du verbe $\left(v_{\mathrm{L}}\right.$ ou $\left.v_{\mathrm{AUXX}}\right)$ décrit à l'instant. L'introduction de la frontière de mot "\#", qui permettait de suivre de près le modèle chomskyen de 1957, n'est donc plus requise, et la contradiction, évitée ${ }^{55}$.

D'autre part, le blocage de toute conjugaison synthétique (sans aux.) pour la plupart des verbes lexicaux basques pourrait trouver son explication dans l'obligation inhérente (donc lexicale) qu'ils auraient de monter dans ASP ${ }^{\circ}$ (la tête d'une projection complément de AuxP, elle-même intermédiaire entre VP et MP dans (61)); il y a peut-être là matière à renouveler la typologie du mouvement des verbes telle qu'elle est développée dans le Programme minimaliste, mais c'est une question qui relève d'un tout autre travail.

Georges REBUSCHI

Centre de recherche en Typologie grammaticale (SYLED, Sorbonne nouvelle / Univ. de Paris III) \& UMR 5478 $<$ rebuschi@ext.jussieu.fr> 


\section{Notes}

1. L'ordre « de base » des éléments pertinents est done assez différent de celui qui est généralement conçu comme canonique sur la base de l'observation en « surface » des morphèmes de certaines formes verbales fléchies; pour une comparaison avec cet ordre « canonique », voir la section 11 .

2. Par un calcul très simple : on a un premier choix binaire (choix du temps "présent" ou "passé" pour FLEX) qui se conjugue à un second choix entre six possibilités (pas de modal ou l'un des cinq mentionnés infra), puis à un troisième choix binaire (sélection ou non des formants du parfait), et enfin à un quatrième (sélection ou non des formants du progressif). On obtient ainsi $2 \times 6 \times 2 \times 2=48 \mathrm{CV}$ différents pour un verbe lexical donné, générés par la simple règle (2), la transformation affixale (voir (4) plus bas) et les possibilités offertes par le lexique spécifique de l'anglais (en ce qui concerne les auxiliaires modaux).

3. Les abréviations et symboles suivants seront utilisés : abs : absolutif; AF, aff : affixe; aff FLEX : affixe flexionnel; all : allocutif; arg : argument; ASP : aspect; AUX : constituant auxiliaire ; aux : (verbe) auxiliaire; CV : complexe verbal; dat : datif; D.E. : déplacement ergatif; DF : dative flag = affixe prédatif; erg : ergatif; fém : féminin; FLEX : flexion verbale (comme constituant); FVF : forme verbale fléchie; hypoth : hypothétique; imp : imperfectif; itér : itératif; masc : masculin; mod : modal ; part : participe; perf : perfectif; pers : personne; $\mathrm{pl}$ : pluriel ; pol : poli; préf : préfixe; prés : présent; prosp : prospectif; sg : singulier; suf : suffixe; T.A. : transformation affixale; TPS, tps : temps; $v$ : verbe; $v_{\text {AUX }}:$ verbe auxiliaire; $v_{\mathrm{L}}$ : verbe lexical ; + : signe de concaténation; \# : frontière de mot; $\sqrt{ }$ : racine (de verbe).

4. (4) est (29.II) dans SS. Le symbole "\#", outre son rôle phonologique (les frontières de mot sont nécessaires à l'assignation de l'accentuation tonique), joue un rôle syntaxique : sa présence dans l'output d'une application particulière de (4) bloque la réapplication du déplacement du suffixe; sans lui, tous les affixes finiraient sur le verbe lexical, comme dans la forme agrammaticale *have be walking-en-s correspondant à la forme réelle ou concrète < has\#been\#walking >. Le symbole "+" ne joue par contre pas d'autre rôle que celui de signaler la concaténation : ce n'est donc pas un "objet 》 (morpho-) syntaxique; par suite, il est automatiquement inséré quand deux morphèmes sont ordonnés, de inême qu'une séquence de deux '+', obtenue dans certains cas par une application de (4), sera automatiquement réduite à un seul.

5. Typologiquement, les Iangues à auxiliaire fléchi accompagné d'un participe verbal sont clairement majoritaires; mais il arrive que l'inverse se produise; ainsi, en albanais (Boneh 1999), les auxiliaires de mode (subjonctif, conditionnel, et... futur) sont invariables, le verbe lexical restant le support de FLEX; en tzeltal, langue maya (Polian 1999), les aux. aspectuels d'accompli et d'inaccompli sont également invariables, le verbe lexical étant, tout comme en leur absence, marqué pour le temps et l'accord. Comme le basque se comporte de ce point de vue comme l'anglais (et le français), je laisserai ce point de côté dans cet article, mais il devrait être clair que de tels phénomènes n'affectent pas nécessairement la hiérarchie sous-jacente a ( 1 a) et ( 1 b) : c'est plutôt la transformation affixale qui est le locus de ces variations, si l'on peut généraliser le développement de la section 10 .

6. Cette prédiction est faite par l'énonciateur; elle est fondamentalement, mais non uniquement, chronologique quand elle est associée aux terminaisons du présent, et plus essentiellement logique dans le cas du conditionnel (quand il ne correspond pas à un « futur dans le passé »).

7. Même si ce dernier est compatible avec des éléments régissants dénotant la visée (comme le verbe aller dans son emploi de futur proche), ou dans les propositions infinitives introduites par pour:

8. Je laisse de côté le choix entre être et avoir comme auxiliaires, mais y reviendrai très brièvement à l'occasion de l'analyse du basque (cf. 5. et 7.1).

9. " $\sqrt{ }$ " indique la racine : c'est bien celle-ci, et non l'infinitif (forme de citation des verbes) qui est introduite par la règle.

10. A vrai dire, la frontière de mot qui suit have ici n'est pas introduite par (4), puisque l'auxiliaire modal n'est pas associé à un affixe quelconque; on peut y remédier en posant l'existence d'un affixe $\emptyset$ associé aux modaux : cet affixe, choisi en même temps que will dans notre exemple, passerait à droite du verbe qui suit (l'auxiliaire have) et déclencherait donc l'apparition de ce \# par application de (4). On verra avec (22), dans la section 5, qu'il y a de bonnes raisons de postuler l'existence d'un affixe post-verbal zéro en basque également.

11. A strictement parler, la seconde application de (4) donne donc une séquence $\langle v+r]+$ ait $+\#+\#\rangle$, avec donc deux frontières de mots à droite, ce qui est sans conséquence. 
12. Ce principe n'était pas connu quand Ruwet a rédigé son livre; cela dit, il n'y discute pas de la formation du futur et du conditionnel, se contentant d'introduire un élément - $r$-dans un arbre syntagmatique sous TPS (notre FLEX) et à gauche de "Prést" (présent) (p. 268).

13. Je segmente les mots orthographiques simples du basque par des points (.): le trait d'union ne sera utilisé que pour isoler la particule suppositive $b a$ - 'si', qui est indépendante des questions étudiées ici.

14. Le - $t$ du préfixe nint-est en fait le - $d$ de nind-dans (9a), dévoisé au contact du $z$ - (une sourde en basque !) du radical $-z(e)$-.

15. Bien distinguer la catégorie "Aux" qui peut comprendre plusieurs éléments, du mot "aux", qui est inanalysable.

16. On notera ici un paradoxe : l'analyse linéaire, et donc morphologique, du CV proposée ici pourrait précéder certains déplacements syntaxiques. Voir la section 11 pour la résolution de ce problème.

17. Voir Rebuschi (1983) pour une description sémantique de ces combinaisons.

18. Le - $n$ final est ici une marque de subordination, et non celle du passé.

19. L'interprétation de plus grande antériorité est typique des dialectes du nord; au sud, c'est le caractère itératif de la deuxième traduction qui prime, comme dans les cas (b) et (c).

20. Plus marqué comme itératif que la forme de (10b').

21. Les verbes dont le part. perf. en est $-n$ ont une forme radicale qui lui est identique.

22. Le fait que la séquence $\langle T U+$ aux $>$ dépende de la présence de la suivante, $\langle$ ASP + aux $>$, est peutêtre dû à ce que l'imperfectif basque (-TZEN + aux), qui est le corrélat morphologique (mais non sémantique) du progressif anglais, est donné par ASP dans ce qui était étiqueté "Aspect. I" dans ( lb).

23. Cette forme est manifestement irrégulière, avec un allomorphe $\emptyset$ pour la racine ; l'analyse de zen en (b) ci-après est quant à elle ambiguë, car le $z$ - initial peut appartenir à la racine (cf. nint.ze.n 'j'étais') ou bien être considéré comme une marque de temps, la racine étant à nouveau - $\emptyset$ -

24. Azkue (1923-25: $§ 771$ ) ajoutait, comme d'autres l'ont fait depuis, un quatrième préfixe, le bedes impératifs de 3e pers. Mais il n'appartient certainement pas au même paradigme, puisque, d'un côté, il est toujours en tête et que, de l'autre, il précède des préfixes absolutifs dans les dialectes du nord (cf. Rebuschi 1984), cf. ikus be.na.za 'qu'il me voie' où la racine de l'aux-za-est précédée de l'abs. na-, qui est à son tour précédé de be-; je laisserai donc ce préfixe de côté dans ce travail.

25. Ou parfois nen-, hen- etc., comme dans (28a', b',c').

26. Les formes indiquées comme "2 pol (i)" sont référentiellement singulières, mais morphologiquement parallèles à celles de la le pers. du pluriel ; la dernière forme est clairement surpluralisée (ajout d'un suffixe - ou -te). Ces faits sont sans importance pour la question débattue.

27. La distribution des affixes (redondants !) marquant spécifiquement le pluriel de l'absolutif est trop irrégulière pour que j'aie quoi que ce soit à en dire ici; on notera que c'est un préfixe, -it-au présent, et - $t$ - au passé, pour 'edun 'avoir', un infixe (-z-) pour eduki 'tenir'(cf. gauzkak 'tu nous tiens'), et le même $-z$ (ou parfois un -de) comme suffixe avec la plupart des autres verbes...

28. On est bien loin de la prétendue théorie de la « non-orientation » du prédicat basque due à Martinet : seules les formes non-fléchies, participiales ou gérondives, sont passibles d'une interprétation valencielle réduite. En tout état de cause, c'est une question qu'un traitement détaillé de la sélection des auxiliaires par les $v_{L}$-ou l'inverse? - devrait prendre en compte.

29. Il est nécessaire de restreindre T.A. à des affixes issus de la catégorie Flexion, si l’on veut éviter que les affixes aspectuels ne passent à gauche des verbes lexicaux.

30. On s'accorde à voir en Azkue (1923-25: § 771) le père de l'hypothèse D.E, ; elle a été reprise par bien d'autres depuis, dont Goenaga (1978: 160), Bossong (1984: 352), Laka (1988, 1993), Albizu (1997) ou Rotaetxe (1998). L'hypothèse de l'« antipassivation » interne à la FVF avancée par Heath (1977) relève de la même démarche.

31. Voir aussi Laka (1993: (63)): "Si la position absolutive est vide, déplacer le clitique ergatif dans cette position." 
32. Le découpage de l'aux. zenion et la glose (légèrement adaptée) - en particulier « $\mathrm{ABS}_{\mathrm{ERG}} 2 \mathrm{sg}$ » sont d'Albizu.

33. Voir aussi Laka (1995, (51), qui découpe étrangement na.u.zu/abslsg-Vaux-erg2pol/, soit 'vous m'avez', en/n-au-zu/, puis nind.u.zu.n/abs 1 sg-Vaux-erg2pol-passé/, 'vous m'aviez'en/n-indu-zu-n/!

34. Gómez \& Sainz (1995: 255) admettent aussi que Ø- devait être originel, mais proposent d'autres sources possibles pour $z-$

35. Voir les poèmes du début du XVII siècle publiés dans Michelena (1964).

36. Ce qui infirme au passage l'hypothèse avancée par Trask (1997: 224), selon qui le haut-navarrais méridional représente une évolution « clairement secondaire et tardive ». Voir aussi Añibarro (1823 [?]), qui signale que les formes guipuzcoanes de son époque avait un (a)n-final optionnel, mais doté d'un contenu sémantique, celles sans suffixe étant traduites par un imparfait (podia $+v_{L}$ etc.), et les autres par un prétérit (pude $+v_{\mathrm{L}} \ldots$ ).

Pour des données plus récentes, voir aussi Azkue (1923-25, § 822) pour un parler biscayen d'Alava qui fonctionnait de la même manière au début du XX siècle, ou encore Gaminde (1994) et Camino (1994), pour des données tout à fait contemporaines dans des parlers respectivement biscayen et aezcoan.

37. On voit ici que l'aoriste pouvait aussi, autrefois, être au passé de l'indicatif (ce temps est d'ailleurs Ie temps ordinaire de la narration dans la trad. du N.T. par Liçarrague, an 1571, dans un dialecte bien éloigné du biscayen). Quant au symbole " $\sqrt{ }$ ", il sert à désigner la racine du verbe concerné.

38. "DF" est pour Dative flag, ou préfixe pré-datif : cet élément apparaît souvent immédiatement devant le suffixe de datif, qui est identique au suffixe ergatif aux deux premières personnes - à la troisième, il y a par contre un segment explicite, $-o$ - cf. (37), qui ne contient pas de référence au datif, et qui n'a donc pas à être modifié.

39. L'absence de segment initial est irrégulière : on attendrait *da.tzait, ce que confirment les formes du passé et de l'hypothétique, zi.tzaidan 'il m'était'et ba-li.tzait 's'il m'était'.

40. La charge de la preuve me semble ici être dans le camp de ceux qui souhaiteraient montrer que l'argument absolutif est plus proche du radical verbal que l'argument datif-voir par ex. Laka (1993: 38) ou Cheng \& Demirdache (1993: 74).

41. Le $h$-initial qu'on rencontre dans certains textes standardisés du Pays basque sud dans la forme h.u.en 'il était (all. : masc)' semble contredire cette affirmation, mais il faut noter que le graphème $h$ ne s'y prononce jamais, que la forme féminine correspondante est h.u.na.n, avec le -na-allocutif clairement post-radical, et qu'en navarro-labourdin, le dialecte contigu au nord-est, les formes sont plus régulières : zu.ka.n, zu.na.n; il faut donc considérer le $h$ - initial de ces deux exemples comme une fantaisie orthographique reposant sur une analyse erronée des faits.

42. On attendrait un $d$ - initial, et c'est le segment qu'on trouve effectivement dans de nombreux dialectes; la spirantisation qui a lieu ici correspond parfois à l'insertion d'un - $i$ - entre le préfixe et le radical, mais les modifications morphonologiques des FVF à affixe allocutif sont trop complexes pour être prises en compte ici.

43. Je préfère "personne" à "accord", malgré i'hypothèse avancée par Oyharçabal (1993) concernant la nature de la marque allocutive.

44. Il est vrai, on I'a vu, que "TPS" en basque peut prendre une valeur hypothétique, donc modale également; mais on a aussi vu que le passé et l'hypoth. ne se différencient qu'à certaines personnes : une forme comme nu à préf. erg. de le p. sg. est donc neutre entre une interprétation temporelle stricio sensu, qui lui sera donnée par le suffixe -en 'je l'avais', et une double interprétation hypothétique, qui lui sera donnée soit par ba- 'si'(banu 'si je l'avais') soit justement par -ke (nuke 'je l'aurais') - sans parler de la réduction, purement morpho-phonologique, de na.u 'il m'a'en n.u/n.ü en bas-navarrais et en souletin. Du point de vue morphologique, l'hypothétique basque est donc bien un sous-cas du temps non-présent/non-actuel, comme indiqué en (34), plutôt qu'une modalité (cf. les emplois dits « modaux 》 du prétérit anglais et de l'imparfait français, déjà mentionnés).

45. On a déjà vu que le - $k$ - initial du suffixe $k$ (a) de 2 e pers. du sg. s'élidait souvent à l'intervocalique; avec un interlocuteur féminin, on aurait eu d.i.que.na.da.

46. Ce qui est un argument de plus pour la thèse soutenue ici, à savoir que la séquence de base est $<v+k e>$, sans intervention de l'affixe datif. 
47. Dans les dialectes du nord, il y a par ailleurs une tendance spontanée à dupliquer l'affixe datif ; par ex., on trouve en souletin zai.ta.da.k, comme forme allocutive (masc.) de zai.t 'il m'est'; en ce qui concerne le labourdin, dans la version des Evangiles de Haraneder (ms. de 1740) « révisée » par Harriet (1855), on trouve dans Mat 14,28 aguin d.ieça.da.çu.t 'ordonnez-moi (que...)', alors que l'original avait simplement mana ça.çu 'ordonnez', sans aucun marque dative, et dans Mat 17,16 ekhar d.ieça.da.çue.t 'apportez-le moi' là ou le texte du siècle précédent avait ekhar dieçada.çue, sans duplication! II est donc possible que l'évolution labourdine soit due à une copie de l'affixe de datif à gauche de -ke plutôt qu'à un mouvement de ce dernier vers la droite. Abondance de biens ne nuit pas.

48. Dans ce dialecte, le DF des verbes tri-actanciels est -ts-, qui se réduit à -s-devant consonne; de plus, on a déjà vu que la voyelle de -ke-y est souvent fermée - et en particulier devant une voyelle, comme ici.

49. Rappelons que seules les FVF sont marquées pour la valence.

50. Si l'on demande d'où vient cette propriété, je dirai simplement que les propriétés de la troisième face des éléments linguistiques (la face grammaticale, « omise » par de Saussure) ont des rapports généralement arbitraires avec les deux autres, cf. par ex. le genre pour les noms à référent inanimé en français,

51. Se rappeler aussi qu'il a été prévu de copier le trait [ ${ }^{2}$ présent] de TPS sur l'affixe déplacé : le choix entre na-et nind-pour abslsg en dépend crucialement.

52. Cette formulation souffre donc des difficultés recensées en 7.2, et ne rend pas compte non plus de l'ordre usuel, même s'il n'est pas standard, <ke+dat $>$ discuté en 9.1. La formule de Laka est reprise par Cheng \& Demirdache (1993). Quant à la formule de Goenaga (1978: 171) : « AUX $\rightarrow$ (asp) $d-+$ pers. (modalité) », elle apparaît plus comme un paquet de traits que comme une véritable concaténation de morphèmes, à quelque niveau de représentation que ce soit.

53. Dans le modèle que je propose, on peut aussi faire des affixes les arguments proprement dits, les SN étant toujours adjoints et donc en position non-argumentale, solution proposée par Jelinek (1984, etc.) pour diverses langues australiennes et amérindiennes.

54. Rappelons que la présentation linéaire de l'AUX anglais dans SS ñ est que la simplification d’une génération effectuée avec des règles de réécriture binaires exposées dans Chomsky (1955). Moins anecdotiquement, la distinction explicite établie ici entre syntaxe et morphologie permet de faire vivre des têtes fonctionnelles (qui légitimeraient par ex. des SN marqués casuellement et fonctionnant comme leur spécificateur) sans qu'il y ait de segment phonologique leur correspondant.

55. Si les mots ne sont pas formés en syntaxe, mais dans le lexique, comme le suggère Chomsky (1995), il n'y a évidemment pas de contradiction non plus. Mais aucune des généralisations proposées ici (ou ailleurs par d"autres) n'aurait de sens. 


\section{Références}

Albizu, Pablo. 1997. 'Generalized Person-Case Constraint : A Case for a Syntax-driven Inflectional Morphology'. In A. Mendikoetxea \& M. Uribe-Etxebarria (eds.), 1- 33.

Allíres, Jacques. 1985. 'Statut et limites du polymorphisme morphologique. Le verbe basque dans la Grammaire cantabrique basque de Pierre d'Urte (i7 12) [Ie partie]'. In J. L. Melena (ed.), 899-919.

Añibarro, Pedro A. 1823 [?]. Gramática bascongada [...]. Ms., ed. par L. Villasante, 1969, in ASUU 3, 3169.

AROTÇARENA, Abbé — 1951. Grammaire basque (dialectes navarro-labourdins). Bayonne : Librairie Le Porche.

AZKUE, Resurrección María. 1923-25. Morfologia vasca (Gramática básica dialectal del Euskera). Facsim., 1969, Bilbao : La Gran Enciclopedia Vasca.

Benventste, Emile. 1959. 'Les relations de temps dans le verbe français'. BSL 54/1, 69-82.

BiCKERTON, Derek. 1981. Roots of Language. Ann Arbor (Mich.) : Karoma.

Bonaparte, Louis-Lucien. 1869. Le verbe basque en tableatx [...]. Londres. Rééd. facsim. in L.-L. B., 1991, Opera Omnia Vasconice, I, Bilbao : Euskaltzaindia (175-442).

BONEH, Nora. 1999. La tête fonctionnelle $M^{\circ}$ en albanais standard d'aujourd hui : les conséquences de sa présence sur l'organisation des catégories fexionnelles. Mémoire de maîtrise, Univ. Paris III.

Bossong, Georg. 1984. 'Ergativity in Basque', Linguistics 22, 341-392.

CAmino, Iñaki. I994. 'A biaburu bat aezkeraren azterketarako'. In R. Gómez: \& J. A. Lakarra (eds.), 59-160.

Cheng, Lisa, \& Hamida Demirdache. 1993. 'External Arguments in Basque'. In J. 1. Hualde \& J. Ortiz de" Urbina (eds.), 71-87.

Chomsky, Noam. 1955. The Logical Structure of Linguistic Theory. Ms. Ed. légèrement abrégée, 1975, New York: Plenum. Rééd., 1985, Chicago: The Univ. of Chicago Press.

Chomsky, Noam. 1957. Syntactic Siructures. Paris/La Haye : Mouton. Trad. fr, 1969, Structures syntaxiques, Paris : Seuil.

Chomsky, Noam. 1995. The Minimalist Program. Cambridge (Mass.) : MIT Press.

Chomsky, Noam, \& Morris Halle. 1968. The Sound Pattern of English. New York : Harper \& Row.

Douglas, William A., Richard W. Etulain, \& William H. Jacobsen, Jr. (eds.). 1977. Anglo-American Contributions to Basque Studies: Essays in Honor of Jon Bilbao. Reno (Nevada) : DRIPSS.

GAmINDE, Iñaki. 1994. 'Plentzia aldeko zenbait ezaugarri morfosintaktiko'. In R. Gómez \& J. A. Lakarra (eds.), 547-551.

Goenaga, Patxi. 1978. Gramatika bideetan. Saint-Sébastien : Erein.

Gómez, Ricardo \& Joseba A. Lakarra (eds.). 1994. Euskal dialektologiako kongresua (Donostian, 1991ko irailak 2-6). Bilbao \& Saint-Sébastien : UPV \& Diputación Foral de Gipuzkoa [Suppléments d' $A S J U$. 28].

Gómez, Ricardo \& Koldo Sainz. 1995. 'On the Origins of the Finite Forms of the Basque Verb'. In J. I. Hualde, J. A. Lakarra \& R. L. Trask (eds), 235-274.

HEATH, Jeffrey. 1977. 'Remarks on Basque Verbal Morphology'. In Douglas et al. (eds.), !93-201 .

Hualde, José Ignacio, Joseba Lakarra \& R[obert] L. Trask (eds.). 1995. Towards a History of the Basque Language. Amsterdam : Benjamins.

HuAdDE, José Ignacio \& Jon Ortiz de Urbina (eds.). 1993. Generative Studies in Basque Linguistics, Amsterdam : Benjamins.

ItHurRY, Abbé —. 1895. Grammaire basque. Dialecte labourdin. Bayonne. Rééd., facsim., 1979, SaintSébastien : Hordago. 
JELINEK, Eloise. 1984. 'Empty Categories, Case, and Configurationality'. Natural Language and Linguistic Theory $2 / 1,39-76$.

LAFITTE, Pierre. 1962. Grammaire basque (navarro-labourdin littéraire). Bayonne: Editions des Amis du musée basque \& Ikas.

LAFON, René. 1944. Le système du verbe basque au XVF siècle. Bordeaux, 2 vol. Rééd. facsim., 1980, Saint-Sébastien: Elkar.

LAKA, Itziar. 1988. 'Configurational heads in inflectional morphology : the structure of the inflected verb forms in Basque'. ASJU 22/2, 343-365.

LAKA, Itziar. 1991. 'Negation in Syntax : On the Nature of Funtional Categories and Projections'. ASJU $25 / 1,65-136$.

LAkA, Itziar. 1993. 'The Structure of Inflection : A case study in Xo syntax'. In J. I. Hualde \& J. Ortiz de Urbina (eds.), $21-70$.

LAKARRA, Joseba. 1996. Refranes y sentencias (1596). Ikerketak ela edizioa. Bilbao : Euskaltzaindia.

LÜDERS, UIrich. 1993. The Souletin Verbal Complex. New Approaches to Basque Morphophonology. Munich : Lincom Europa.

MANANDISE, Esméralda. 1998. Evidence from Basque for a New Theory of Grammar. New York: Garland.

Melena, José L. (ed.). 1985. Symbolae Ludovico Mitxelena Septuagenario Oblatae. Vitoria : Universidad del Pais Vasco.

Mendikoetxea Amaya \& Myriam Uribe-Etxebarria (eds.). 1997. Theoretical Issues at the MorphologySyntax Interface. Bilbao \& Saint-Sébastien : UPV \& Diputación Foral de Gipuzkoa [Suppléments d'ASJU, 40].

Michelena, Luis. 1964. Textos arcaicos vascos. Madrid : Minotauro.

Mizprratzéguy, Fenaille. 1936. Dictiomaire français-basque. Grammaire. Sans indication d'éditeur : ouvrage imprimé à Paris.

OYHARCABAL, Beñat. 1993. 'Verb Agreement with Non-Arguments : On allocutive agreement'. In J. Hualde \& J. Ortiz de Urbina (eds.), 89-1 14.

POLIAN, Gilles. 1999. (Morpho-)Syntaxe du verbe tzeltal. Mémoire de DEA, Univ. Paris IIr.

REBUSCHI, Georges. 1983. 'Enoncé et formes hypothétiques en basque contemporain'. Verbum 6/3, 343361. Rééd. in G. R., 1997, Essais de linguistique basque (Bilbao \& Saint-Sébastien : UPV \& Diputación Foral de Gipuzkoa [Suppléments d'ASJU, 35]), 139-154.

Rebuschi, Georges. 1984. Structure de l'énoncé en basque. Paris : SELAF.

Ruk (DE), Rudolf P. G. 1985. 'Un verbe méconnu'. In J. L. Melena (ed.), 92 L -935.

RotaetXe Amusategi, Karmele. 1998. 'Estructura distribucional de la formas finitas en euskera'. In I. Turrez, A. Arejita \& C. Isasi (eds), Sfudia Philologica, In Honorem Alfonso Irigoien (Bilbao: Université de Deusto), 207-224.

RuweT, Nicolas. 1968. Introduction à la grammaire générative. Paris : Plon.

TRASK, R[̨obert] L. 1977. 'Historical Syntax and Basque Verbal Morphology : Two Hypotheses'. In Douglas et al., 203-217.

TRASK, R[obert] L. 1977. The History of Basque. Londres ; Croom Helm. 